Bangladesh Journal of Neuroscience 2015; Vol. 31 (2): 94-101

\title{
Intraventricular Tumor: An Analysis of 18 Cases
}

\author{
SHAMSUL ALAM ${ }^{1}$, A.N. WAKIL UDDIN², MASHIUR RAHMAN MAJUMDER ${ }^{3}$, \\ MD MOTASIMUL HASAN. ANIS AHMED ${ }^{5}$
}

\begin{abstract}
:
Objective: To describe the transcallosal and transcortical approach to deal with intraventricular tumors. Methods: Details of the transcallosal and transcortical approach to intraventricular tumors of the lateral and third ventricles were presented. Results: Intraventricular tumors are ideal indications for microscopic neurosurgery. They often cause cerebrospinal fluid (CSF) pathway obstruction, resulting in ventricular dilatation. The general principle of removal of intraventricular tumors was interruption of the blood supply to the tumor and subsequent tumor debulking. In general, a piecemeal resection was performed; however, in some tumors such as meningioma, it was possible to detach the lesion from the surrounding brain tissue and remove it in toto. When the tumor found in the anterior part of the third ventricle, the craniotomy was done at the coronal suture. When the tumor was located in the posterior part, the entry craniotomy was selected more anteriorly in order to pass the foramen of Monro in a straight line. Conclusion: Intraventricular tumors and related CSF pathway obstructions can be safely and effectively treated with micro neurosurgical techniques, either by transcallosal or transcortical approach. The aim should be the total extraction of the tumor with minimum damage and the chosen operative corridor should optimize tumor access and the protection of vulnerable neurovascular structures. Lateral ventricle tumors can be removed via transcortical approach when having hydrocephalus which provides a wider and more direct approach to the tumor than the transcallosal one. It allows the surgeon to achieve good functional outcome and maximum excision of the tumor. Transcallosal is an excellent midline exposure with preserving the callosomerginal and pericallosal arteriesto the midline tumor of lateral and $3^{\text {rd }}$ ventricles.
\end{abstract}

Key Word: Intraventricular Tumor, Meningioma, Central Neurocytoma.

Introduction:

Intraventricular neoplasms are uncommon, representing just $1-10 \%$ of all CNS tumors. Lateral ventricular neoplasms are rare and account for half of all intraventricular tumors in adults and one quarter of those in children. While these neoplasms are easily detected with computed tomography (CT) and magnetic resonance imaging $(\mathrm{MRI})^{1,2}$.

The ventricles are surrounded by a layer of ependymal cells and a subependymal plate formed by glial cells. Such layers give origin to ependymomas, subependymomas and subependymal giant cell astrocytomas. Such a lining and the septum pellucidum that is located between the corpus callosum and the fornix, separating the lateral ventricles, also give origin to central neurocytoma, a unique glial neuronal tumor of the ventricular systems ${ }^{2,3}$.

Tumors are more frequently found in the posterior portion of the lateral ventricles, but their location may vary according to the type of tumor. Choroid plexus papillomas occur mainly in children, with predilection for the lateral ventricles in this age range, while in adults it usually is more frequently

1. Assistant Professor, Department of Neurosurgery, Bangabandhu Sheikh Mujib Medical University, Dhaka.

2. Research Assistant, Department of Neurosurgery, Bangabandhu Sheikh Mujib Medical University, Dhaka.

3. Registrar, Department of Neurosurgery, Comilla Medical College, Comilla.

4. Resident. Department of Neurosurgery, Bangabandhu Sheikh Mujib Medical University, Dhaka.

5. Medical Officer, Department of Neurology, , Bangabandhu Sheikh Mujib Medical University, Dhaka. 
found in the fourth ventricle. Ependymomas are most frequent in the posterior fossa in children, and in adults they are generally supratentorial $\left.\right|^{3,4}$.

Tumors of the lateral ventricle can be removed via two major approaches, either through the transcallosal or transcortical route. The purpose of this study is to discuss the techniques and outcomes in transcallosal and transcortical surgery of tumors located in the lateral ventricle and $3^{\text {rd }}$ ventricle ${ }^{4,5}$.

The transcallosal route is preferred by some neurosurgeons for a variety of reasons. The transcallosal approach may decrease the risk of postoperative seizures and functional deficits. It is recognized that the transcallosal route to the ventricles can be safely performed to excise lesions in the ventricular body, anterior horn, atrium and $3^{\text {rd }}$ ventricles ${ }^{5,6}$.

The transcortical approach to the lateral ventricles is a simple and attractive alternative to the transcallosal approach for many deep-seated tumors. It has the advantage of simplicity. Tumors located in certain regions of the lateral ventricle, specifically the temporal horn or atrium, are often more safely and easily approached through a cortical incision. Transcortical approach provides superior working space and more flexibility in traversing the lateral ventricle ${ }^{5,6}$.

We used either transcallosal or the transcortical routes for the excision of lateral ventricular masses. This article is based, in part, on our recent 5-year experience with excising mass lesions in the lateral ventricle and $3^{\text {rd }}$ ventricle via the transcortical and transcallosal route.

\section{Relevant Surgical Anatomy:}

An appreciation of the relevant anatomy is extremely helpful when performing transcortical and transcallosal approaches to the lateral ventricles.

The lateral ventricles are paired C-shaped structures that wrap around the ipsilateral thalamus. Each ventricle is divided into five sections: anterior horn, body, temporal horn, atrium, and occipital horn. Each of these five sections has a roof, a floor, and a medial and lateral wall. ${ }^{7,8}$ ( Fig-1).

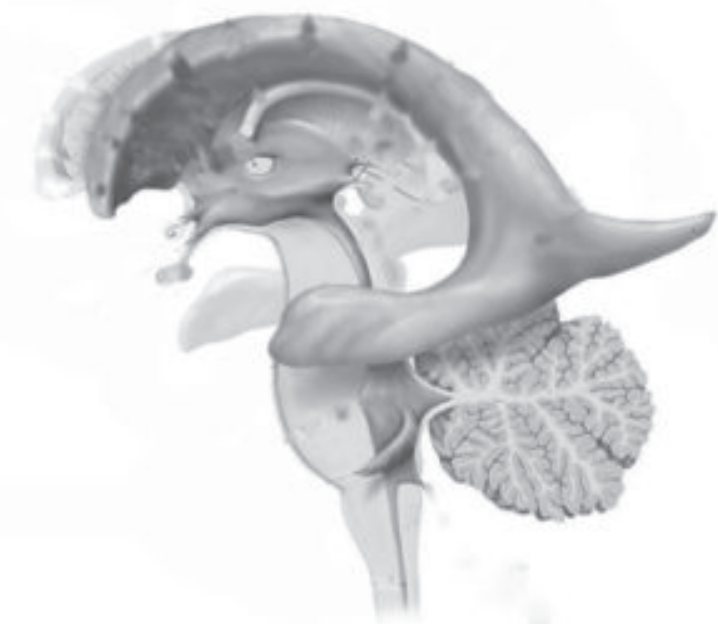

Fig.-1: Shows the normal anatomy of ventricular system.

The walls of the lateral ventricles are formed by the thalamus, septum pellucidum, corpus callosum and its radiations, caudate nucleus, and the fornix. The caudate nucleus is also an almond-shaped structure that forms the lateral wall of the body, anterior lateral wall of the atrium, and roof of the temporal horn. The fornix starts as the fimbria of the hippocampi in the medial temporal horn and runs along the thalamus in the anterior wall of the atrium. After giving off commissural fibers, it continues in the inferior medial wall of the body. Finally, it forms the superior and anterior borders of the foramen of Monro ${ }^{8}$.

Although it provides limited access to tumors in the posterior trigone, temporal horn or superior frontal horn, the transcallosal approach to the lateral and third ventricles can provide maximal exposure to tumors arising body of lateral ventricle and foramen Monro. Patients harboring tumors arising in those locations are best managed by transcortical approaches. Each of these surgical options has its own set of potential complications ${ }^{8,9}$.

The primary arteries to the choroid plexus are the anterior and posterior choroidal arteries, the branches of which provide the vascular supply to tumors in this region. Understanding the course of the arteries helps the surgeon choose an approach for each lesion and thus permits early control, when possible, of the feeding vessels ${ }^{9}$. 
The anterior choroidal artery arises from the internal carotid artery, distal to the posterior communicating artery. It leaves the anterior incisural space and enters the lateral ventricle through the choroidal fissure, coursing posteriorly to lie near the lateral posterior choroidal artery. The anterior choroidal artery generally supplies the choroid plexus in the temporal horn and atrium. Because the choroidal arteries pass through the choroidal fissure, opening this fissure early will facilitate proximal control of the feeding vessels ${ }^{9}$.

The posterior choroidal arteries are grouped into lateral and medial divisions. The lateral posterior choroidal artery is comprised of one to six branches, which arise in the ambient and quadrigeminal cisterns from the PCA. These branches then pierce the ventricle and pass around the pulvinar and through the choroidal fissure at the level of the crus of the fornix to supply the choroid plexus in the posterior temporal horn, atrium, and body of the ventricles. The medial posterior choroidal arteries arise as one to three branches from the PCA in the interpeduncular and crural cisterns. These arteries circumnavigate the mid-brain and move to the pineal gland to enter the roof of the third ventricle. This vessel then passes in the velum interpositum, between the thalmi, adjacent to the internal cerebral veins. The medial posterior choroidal arteries travels through the velum interpositum (tela choroidea), sending inconstant branches to the lateral ventricle through the choroidal fissure and foramen of Monro. The medial posterior choroidal artery supplies the choroid plexus in the roof of the third ventricle and sometimes the choroid plexus of the lateral ventricle ${ }^{9}$.

The veins are useful as landmarks to direct the surgeon to the foramen of Monro, especially in cases in which hydrocephalus is present. There are many important veins composing the lateral and medial groups, but perhaps the best known for surgical and angiographic orientation is the thalamostriate vein. The thalamostriate vein courses from the lateral wall of the body of the ventricle through the sulcus between the caudate and thalamus toward the foramen of Monro, where it forms the venous angle with an acute posterior turn to empty into the internal cerebral veins. The veins, which drain the frontal horn and usually anterior lateral ventricle, drain into the internal cerebral vein as it travels in the roof of the third ventricle within the velum interpositum. The veins in the temporal horn drain into the basal vein of Rosenthal (basal vein) as it passes through the ambient cistern. Veins from the atrium and occipital horn drain into the basal internal cerebral veins as well as the vein of Galen ${ }^{9,10}$.

There are several options for accessing the third ventricle via the transcallosal approach. The foramen of Monro provides access to the anterior third ventricle; however, lesions in the middle and posterior third ventricle require an alternative exposure. An expanded foramen does not routinely need to be enlarged by sacrifice of a fornix. If a lesion located within the third ventricle can be decompressed and delivered into the foramen, then this is the most appropriate use of this approach ${ }^{10}$.

A common approach to the middle and posterior third ventricle is through the choroidal fissure into the velum interpositum. This space is defined laterally by the insertion of the choroid plexus, superiorly by the fornix, and inferiorly by the dorsomedial thalamus. The choroid plexus can be removed along its insertion in the choroidal fissure ${ }^{10}$.

The fornix is then gently elevated along with the internal cerebral vein, which opens the velum interpositum. The tumor is decompressed into the area of exposure and mobilized away from the ependymal surface of the ventricle ${ }^{10}$.

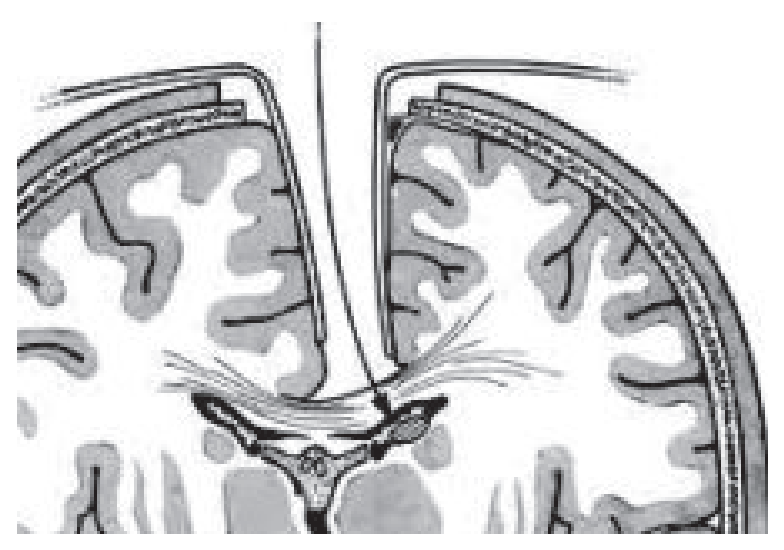

Fig.-2: Picture of transcallosal approach. 
Methods and Materials:

An experience with 18 consecutive lateral and $3^{\text {rd }}$ ventricular tumors resected via the transcortical and transcallosal route, over a 5-year period, was presented. The risks, complications, and outcomes of this surgical series, as well as those reported in the literature, we discussed.

Following tumor resection by piecemeal fashion, it is important to ensure complete hemostasis. To prevent delayed ventricular obstruction, care is taken to irrigate blood that may have pooled in the lateral and third ventricles. The ventricles are filled with warm saline at the end of the surgery,

to remove air that may have become trapped. A ventricular catheter is left in the lateral ventricle for approximately 96 hours postoperatively to monitor intracranial pressure and to demonstrate that the ventricular system is patent. It is routine to obtain a CT scan on the 1st postoperative day to evaluate the extent of the tumor resection and to check for obstruction. The catheter can be removed at the bedside prior to mobilization of the patient to ward or before discharge.

\section{Symptomatology:}

The most common symptoms and signs of intraventricular tumor were intracranial hypertension, hydrocephalus and hemiparesis. Hydrocephalus are common in those tumor which were located at the foramen of Monro. Parinaud syndrome and diabetes insipidus are common in patients harboring a third ventricle tumor.

\section{Results:}

We did surgery in 18 cases of intraventricular tumor. Among them 10 were male and 8 were female (Table-I).

Table-I

Shows the Sex of patients ( N-18)

\begin{tabular}{lcc}
\hline Sex & No. of Patient & Percentage \\
\hline Male & 10 & $55.55 \%$ \\
Female & 8 & $45.45 \%$ \\
\hline Total & 18 & $100 \%$ \\
\hline
\end{tabular}

Ages varies from 10-65 years. Mean age was 33 years (Table II).
Table-II

Shows the age of patients ( N-18)

\begin{tabular}{lcc}
\hline Ages & No. of Patient & Percentage \\
\hline$>10$ & 1 & $5.56 \%$ \\
$10-20$ & 2 & $11.11 \%$ \\
$21-30$ & 4 & $22.22 \%$ \\
$31-40$ & 7 & $38.88 \%$ \\
$41-50$ & 1 & $5.56 \%$ \\
$51-60$ & 2 & $11.11 \%$ \\
$<60$ & 1 & $5.56 \%$ \\
Total & 18 & $100 \%$ \\
Mean Age & 33 Years & \\
\hline
\end{tabular}

Most of the tumor well located in the body of lateral ventricle. Only 3 cases were anterior $3^{\text {rd }}$ ventricular tumor ( Table III)

Table III

Location of Tumor( $\mathrm{N}-18)$

\begin{tabular}{lcc}
\hline Location & No. of cases & Percentage \\
\hline Body of Lateral ventricular & 9 & $50.00 \%$ \\
Trigone of Lateral Ventricle & 3 & $16.66 \%$ \\
Foramen monro & 3 & $16.66 \%$ \\
$3^{\text {rd }}$ Ventricle & 3 & $16.66 \%$ \\
\hline Total & 18 & $100 \%$ \\
\hline
\end{tabular}

The transcallosal (44.44\%) or transcortical (55.56\%) approach was used depending upon the ventricular dilatation, tumor location and proximity of the tumor in relation to approach ( Table-IV)

Table-IV

Table of Approach ( N-18)

\begin{tabular}{lcc}
\hline Name of approach & No of cases & Percentage \\
\hline Transcortical & 10 & $55.56 \%$ \\
Transcallosal & 8 & $44.44 \%$ \\
\hline Total & 18 & $100 \%$ \\
\hline
\end{tabular}

Gross total removal was done in $13(72.23 \%)$ cases, subtotal in $3(16.67 \%)$ cases, near total in one case $(5.55 \%)$ and partial removal was done in one case (5.55\%). (Table-V) 
Table-V

Shows the Extent of Tumor Removal( $N-18)$

\begin{tabular}{lcc}
\hline $\begin{array}{l}\text { Extent of Tumor } \\
\text { Removal }\end{array}$ & No. of Patient & Percentage \\
\hline Gross Total & 13 & $72.23 \%$ \\
Sub Total & 3 & $16.67 \%$ \\
Near Total & 1 & $5.55 \%$ \\
Partial & 1 & $5.55 \%$ \\
\hline Total & 18 & $100 \%$ \\
\hline
\end{tabular}

At surgery, three neurocytomas appeared as originating from the septum pellucidum and another appeared as arosed from the head of the caudate nucleus. Three cases of meningioma are arosed from choroid plexus. All three ependymomas appeared as originating from the septum pellucidum and one SEGA from the head of the caudate nucleus. Two cases of pilocytic astrocytoma arised from thalamus. (Table VI)

Table-VI

Shows the Histopathology ( N-18)

\begin{tabular}{lcc}
\hline Histopathology & No. of Patient & Percentage \\
\hline Neurocytoma & 4 & $22.23 \%$ \\
Meningioma & 3 & $16.68 \%$ \\
Lateral Ventricular & 3 & $16.68 \%$ \\
Ependimoma & & \\
$\begin{array}{l}\text { Pilocytic astrocytoma } \\
\text { Epidermoid }\end{array}$ & 1 & $5.55 \%$ \\
$\begin{array}{l}\text { Subependymal Giant Cell } \\
\text { astrocytoma }\end{array}$ & 1 & $5.55 \%$ \\
Thirdventricular tumor & 1 & $5.55 \%$ \\
Ependimoma & & $5.55 \%$ \\
Thalamic glioma & 2 & $11.11 \%$ \\
Choroid plexus papilloma & 1 & $5.55 \%$ \\
Adenomas carcinoma & 1 & $5.55 \%$ \\
Cyst & & \\
\hline Total & 18 & $100 \%$ \\
\hline
\end{tabular}

Radiation therapy was given to a patients who had adenoid carcinoma cyst, of lateral ventricle which was recurred one year after surgery.

Seizure following surgery occurred in 2 cases( $28.57 \%$ ). Meningitis was developed in 3 cases ( $42.85 \%$ ) which was improved by injection Meropenum.

VP Shunt was done by 2 cases $(28.57 \%$ ) (Table VII).
Table-VII

Table of Complication ( $\mathrm{N}-18)$

\begin{tabular}{lcc}
\hline Name of complication & No of cases & Percentage \\
\hline Meningitis & 3 & $42.85 \%$ \\
VP Shunt & 2 & $28.57 \%$ \\
Seizure & 2 & $28.57 \%$ \\
\hline Total & 7 & $100 \%$ \\
\hline
\end{tabular}

One year after surgery, $77.78 \%$ of patients had good functional outcome. ( Table VIII )

Table-VIII

Table of Glasgow outcome following surgery $(N-18)$

\begin{tabular}{lcc}
\hline Functional outcome & No of cases & Percentage \\
\hline Good Recovery & 14 & $77.78 \%$ \\
Moderate Disability & 2 & $11.12 \%$ \\
Severe disability & 1 & $5.55 \%$ \\
Vegetative & 1 & $5.55 \%$ \\
Death & 0 & 0 \\
\hline Total & 18 & $100 \%$ \\
\hline
\end{tabular}

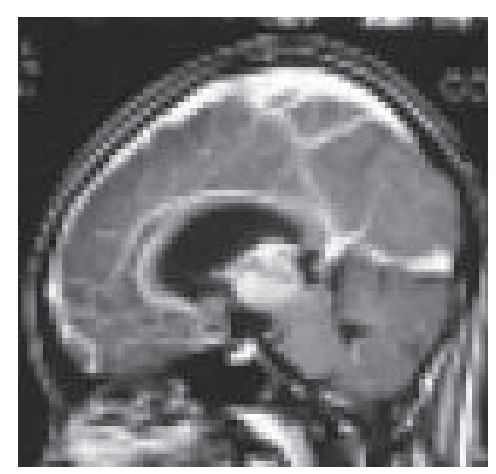

Fig.-3: Preoperative picture of $3^{\text {rd }}$ ventricular ependyomoma.

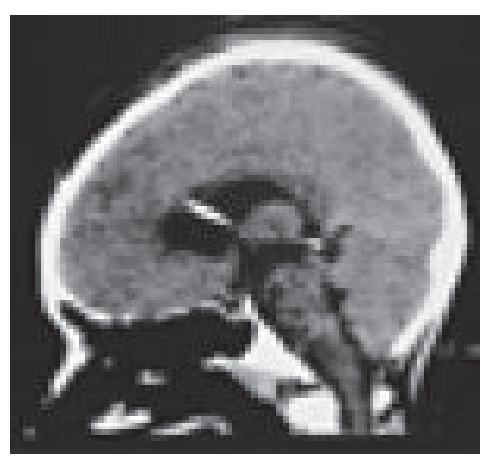

Fig.-4: Postoperative picture of $3^{\text {rd }}$ ventricular ependyomoma done by transcallosal approach. 


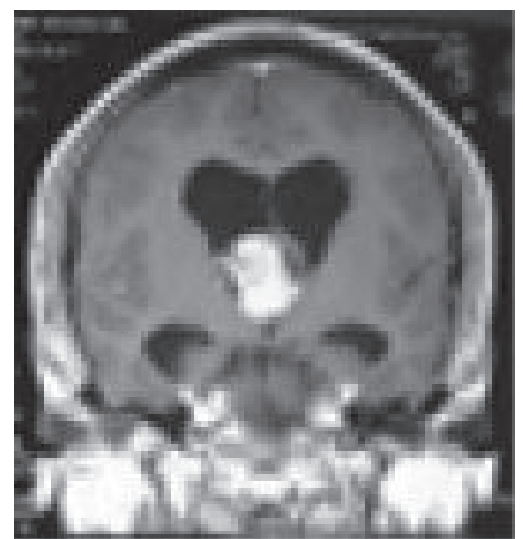

Fig.-5: Preoperative picture of Pilocytic astrocytoma.

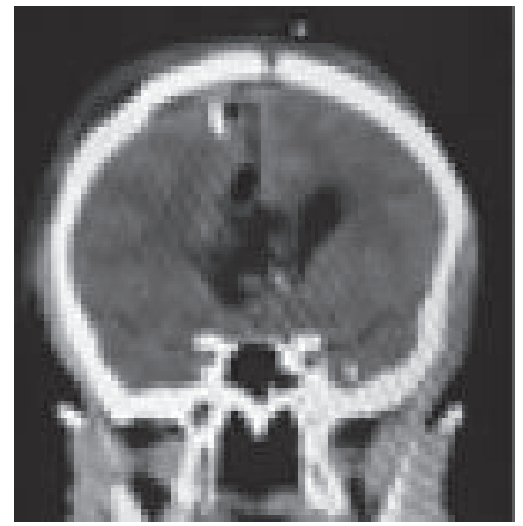

Fig.-6: Postoperative picture of Pilocytic astrocytoma done by transcallosal Approach.

\section{Discussion:}

The incidence of cortical incision-related postoperative epilepsy is hard to determine because there are many factors that can contribute to a seizure disorder, including the tumor histological type, presence of preoperative seizures, presence of residual tumor, subdural hygroma, and electrolyte imbalance. Any patient who undergoes a craniotomy or has his/her cortex broached is at risk for postoperative seizures. Rates of postoperative seizures have been noted to be as low as $19 \%$ in a recent study and as high as $75 \%$ in a study reported in the pre-microsurgical age.It is difficult to isolate surgical technique accurately in the various studies and arrive at an incidence of transcortical incisioninduced postoperative epilepsy ${ }^{7}$. In our recent series at 1 -year follow up, we found an incidence of $22.22 \%$ of persistent postoperative epilepsy that required medication.

Postoperative neurological deficits include hemiparesis and language deficit. A mild to moderate hemiparesis is not uncommon when a large trigone or ventricle body tumor is excised via a middle fossa transcortical approach. Most weakness is presumably the result of retraction pressure and will resolve; however, the incidence of permanent motor loss has been reported to be as high as 30\% in some series ${ }^{12}$. However we have found $11.11 \%$ hemiparesis in all 18 cases.

Cognitive deficits and personality disorders are much more difficult to quantitate, especially if preoperative neuropsychological studies are not conducted. It is wise to leave tumor, especially if it is attached to critical structures such as the basal ganglia or fornices, and particularly if the lesions is benign ${ }^{11}$.

Subdural hygroma formation is a well-recognized problem, especially in patients in whom an enormous tumor is associated with ventriculomegally. This is especially true in pediatric series in which large choroid plexus neoplasms and hydrocephalus are treated. The cortical surface may fall or pull away from the dura and create a hematoma or hygroma, which may eventually require the placement of a shunt. In their 1989 series of transcortical approaches to intra- and periventricular tumors in 38 patients, Tanaka, et al found that persistent subdural fluid accumulations developed in $24 \%$ of the patients; $11 \%$ required surgical treatment. Patients with preoperative hydrocephalus and those who underwent a transfrontal approach were at greatest risk. Filling the ventricles with sterile saline or lactated Ringer solution before the completion of the surgery and leaving both subdural and intraventricular catheters in place may reduce the incidence of hygroma and hydrocephalus ${ }^{11}$.

Postoperative ventriculomegally is common complication. The number of patients requiring a shunt will vary based on different factors. Approximately 10 to $50 \%$ of patients will ultimately require CSF diversion. We did 2 VP shunt( 28.57 $\%)$ out of 18 cases in our series.

All patients may suffer some form of meningeal irritation caused by the presence of blood products 
in the CSF. This type of chemical meningitis can be treated by administering analgesic agents and a tapered course of steroid medication ${ }^{12}$. In our series we report rates of infection is 3 cases. We have 3 cases $(42.85 \%$ ) of pyogenic meningitis which was controlled by injection Meropenem.

Prior to the advent of microsurgery techniques, the mortality and morbidity rates were higher than those of the present day. In early studies the some authors reported mortality rates as high as $75 \%$, mostly because result of intraoperative hemorrhage or cerebral edema.In the more modern series the authors have recorded mortality rates far lower than $10 \%$. The deaths in the microsurgical era are usually secondary to catastrophic postoperative hemorrhage or pulmonary emboli. In our current study we had only one mortality due to intraventricular massive hemorrhage ${ }^{13}$.

As a general principle, the number of veins sacrificed during a transcortical approach should be kept to a minimum. Sacrifice of both deep and superficial veins can cause inconstant deficits, which have been reported as severe by some surgeons. This is a controversial neurosurgical issue. The variation in outcome may be the result of differences in individual anatomy. Dandy claimed that one internal cerebral vein can be sacrificed without effect, and, occasionally, even the great vein of Galen has been ligated without causing death. Other surgeons have found this observation to be incorrect. We made every effort to avoid sacrificing any vein unless it clearly compromises access to the ventricular mass but yet one cortical cerebral vein was injured which leads to hemorrhagic transformation of venous infracts.

All patients was monitored in post-operative setting for at least 24 hours and should undergo noncontrast CT scanning within 72 hours to assess ventricular size and tumor residual ( Figure 4,6). Repeating the neuroimaging study in a week is useful for assessing the presence of extra cerebral fluid collections. It is useful to remove the external catheters/drains as soon as possible and to mobilize the patient. Patients with persistent ventriculomegaly or subdural hygroma are monitored longer and receive antibiotic therapy for as long as a drain is in place. Adequate levels of anticonvulsant agents are monitored at regular intervals and electrolyte imbalance is aggressively treated ${ }^{13,14}$.

The transcortical technique makes it possible to resect lesions in each of the five regions of the lateral ventricle. It provides superior microsurgical working space and flexibility for maneuvering within the lateral ventricle. The key to a successful transcortical approach is an understanding of the functional anatomy of eloquent cortex to be broached, the location of the lesion, and its vascular supply. A clear understanding of the advantages and limitations of the transcortical approach makes performing this procedure for resection of large lesions in the ventricle both safe and effective. The majority of the patients in this series $(77.78 \%)$ had a good outcome, returning to baseline functional status and suffering minimal morbidity. In the microsurgical era, transcortical surgery-related postoperative morbidity and outcome are dependent more on tumor histological type and site of origin than on approach ${ }^{14}$.

The transcallosal approach to the lateral and third ventricle lesions offers an important surgical option (Figure 3,5). There are several advantages to this approach over the transcortical route. With appropriate brain relaxation and minimal retraction, the surgeon can reduce the risk of brain injury. Care to preserve medial draining veins prevents venous infarction. Partial sectioning of the callosum leads to minimal long-term consequences unless additional brain injury is induced. Because this approach is not commonly used in the average neurosurgical practice, it is important to review the anatomy and to pay careful attention to the techniques that optimize a favorable outcome ${ }^{14}$.

Transcallosal surgery carries a reduced risk of postoperative seizures, porencephalic cyst formation, and subdural hygroma formation compared with transcortical surgery.Limited exposure of the posterior lateral ventricle and the apex of the frontal horn, however, reduces the utility of the transcallosal approach for lesions arising in these regions ${ }^{14}$. 


\section{Conclusion:}

Intraventricular tumors and related CSF pathway obstructions can be safely and effectively treated with micro neurosurgical techniques, either by transcallosal or transcortical approach. The aim should be the total extraction of the tumor with minimum damage and the chosen operative corridor should optimize tumor access and the protection of vulnerable neurovascular structures. Lateral ventricle tumors can be removed via transcortical approach when having hydrocephalus which provides a wider and more direct approach to the tumor than the transcallosal one. It allows the surgeon to achieve good functional outcome and maximum excision of the tumor. Transcallosal is an excellent midline exposure with preserving the callosomerginal and pericallosal arteries to the midline tumor of lateral and $3^{\text {rd }}$ ventricles.

\section{References:}

1. Furie D, Provenzale J. Supratentorial ependymomas and subependymomas: CT and MR appearance.J Comput Assist Tomogr 1995; 19:518-526.

2. Scheithauer B. Symptomatic subependymoma: report of 21 cases with review of the literature.J Neurosurg 1978; 49:689-696.

3. Rawlings CE, Giangaspero F, Burger PC, Bullard DE. Ependymomas. Aclinicopathologic study. Surg Neurol 1988; 29:271-81.

4. Anderson RC, Ghatan S, Feldstein NA: Surgical approaches to tumors of the lateral ventricle. Neurosurg Clin N Am 2003;14: 509525.

5. Apuzzo MJ, Amar AP: Transcallosal interforniceal approach, in Appuzzo MLJ (ed):
Surgery of the Third Ventricle, ed 2. Baltimore: Williams \& Wilkins, 1998; 421-452

6. D'Angelo VA, Galarza M, Catapano D, et al: Lateral ventricle tumors: Surgical strategies according to tumor origin and development - $\mathrm{A}$ series of 72 cases. Neurosurgery .2005;56 (Suppl 1):36-45.

7. Ellenbogen RG: Transcortical surgery for lateral ventricular tumors. Neurosurg Focus.2001; 10(6):1-13.

8. Kasowski A, Pipmeier JM: Transcallosal approach for tumors of the lateral and third ventricles. Neurosurg Focus 2001; 10:1-5.

9. Rhoton AL: The lateral and third ventricle. Neurosurgery 2002:51(Suppl 1):207-71.

10. Koeller KK, Sandberg GD. From the archives of the AFIP. Cerebral intraventricular neoplasms: radiologic-pathologic correlation. RadioGraphics 2002; 22:1473-1505

11. Tanaka $\mathrm{Y}$, Sugita $\mathrm{K}$, Kobayashi $\mathrm{S}$, et al: Subdural fluid collections following transcortical approach to intra or paraventricular tumors. Acta Neurochir 99;1989:20-25

12. Santoro A, Salvati M, Frati A, et al: Surgical approaches to tumors of the lateral ventricles in the dominant hemisphere. J Neurosurg Sci 2002;46:60-65.

13. Suh DY, Mapstone T:Pediatric supratentorial intraventricular tumors. Neurosurg Focus 10 E4,2001;19

14. Yasargil MG, Abdularauf SI: Surgery of intraventricular tumors. Neurosurgery 2008;62 (Suppl. 3)1029-41. 